\title{
TRICOBEZOAR GASTRO-DUODENO-JEJUNAL: ABORDAGEM VIDEOLAPAROSCÓPICA
}

\section{GASTRIC-DUODENUM-JEJUNE TRICHOBEZOAR: VIDEOLAPAROSCOPIC MANAGEMENT}

\author{
Antonio Carlos Bartolomucci, TCBC-SP ${ }^{1}$ \\ Alexandre Marotta, TCBC-SP ${ }^{2}$ \\ Edgar Meante dos Santos ${ }^{3}$
}

\section{INTRODUÇÃO}

Bezoares são coleções gástricas de materiais não digeridos, ocasionalmente se estendendo ao intestino, os quais podem ser classificados em quatro categorias: a) Fitobezoar (fibras vegetais); b) Tricobezoar (fios de cabelo); c) Bezoares por medicamentos (comprimidos ou massas de drogas semilíquidas); d) Miscelânea de material (argila, pedra etc.) ${ }^{1}$. Descreveremos um caso de tricobezoar que, pelas suas dimensões (gastro-duodeno-jejunal) e método de tratamento, merece uma análise frente aos dados de literatura.

\section{RELATO DO CASO}

Paciente de 14 anos, sexo feminino, com alopécia há 18 meses e sintomatologia digestiva alta com perda de peso, há seis meses. Em tratamento psiquiátrico há dois anos e, somente há um mês, com comprovação de tricofagia. Os dados clínicos eram de estado geral regular, descorada $+/ 4+$ e massa pétrea em epigástrio e hipocôndrio direito. A endoscopia digestiva alta revelou tricobezoar em todo lume gástrico. Optou-se por cirurgia videolaparoscópica, com acesso umbilical (10mm), em hipocôndrio esquerdo (10mm), e em hipocôndrio direito e paraxifóidea direita $(5 \mathrm{~mm})$.A laparoscopia revelou esteatose hepática e tricobezoar gastro-duodeno-jejunal (Figura 1).

Realizada gastrotomia de $10 \mathrm{~cm}$, longitudinal (corpo-antro); colocação intracavitária de saco plástico para resíduo cirúrgico (coletor de suco

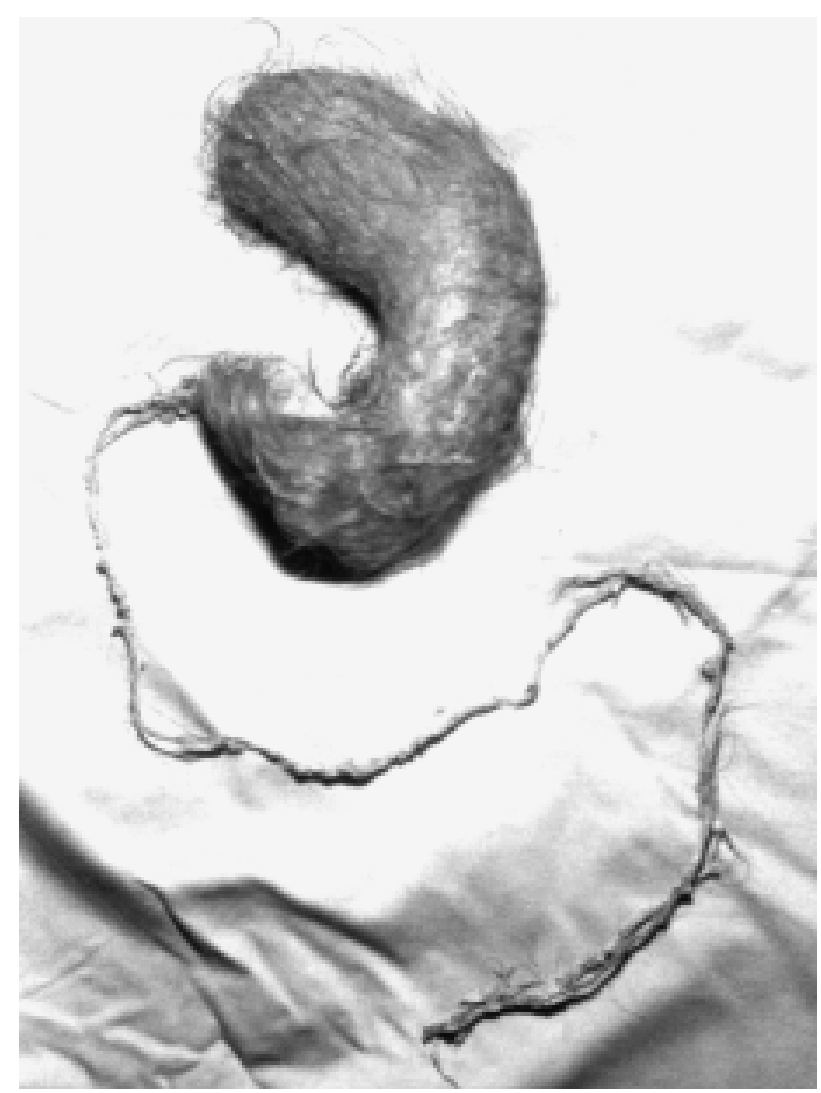

Figura 1 - Tricobezoar Gastro-Duodeno-Jejunal.

gastroentérico); posicionamento do saco coletor com o resíduo (tricobezoar gastro-duodeno-jejunal) na pelve; gastrorrafia em dois planos contínuos $\left(\right.$ Vycril $^{\circledR}$ 3-0 e Ethibond ${ }^{\circledR}$ 2-0); mini-Pfannenstiel para retirada do resíduo cirúrgico.

1. Prof. Titular da Disciplina de Cirurgia Geral - Dpto. Ciências Médicas - UNITAU.

2. Prof. Assistente da Disciplina de Cirurgia Geral - Dpto. Ciências Médicas - UNITAU.

3. Residente de Cirurgia Geral - Hospital Universitário de Taubaté - UNITAU.

Recebido em 31/01/2003

Aceito para publicação em 02/09/2003

Trabalho realizado no Hospital Universitário de Taubaté - UNITAU. 
A alta hospitalar se deu em $48 \mathrm{~h}$. No $8^{\circ}$ P.O. apresentou dor abdominal, febre, edema maleolar bilateral, drenagem purulenta nas portas paraxifoidea e hipocôndrio direito e secreção sero-sanguinolenta em miniPfannenstiel. RX tórax: pequeno derrame costofrênico esquerdo. USG: líquido livre na cavidade abdominal.

Realizou-se reintervenção através da incisão de Pfannenstiel, tendo sido evidenciado líquido seroso sem fibrina $(500 \mathrm{ml})$ na cavidade e integridade da cirurgia gástrica. Realizada drenagem sob vácuo (flancos e pelve). Apresentou boa evolução e alta hospitalar no $7^{\circ}$ P.O. Controle clínico até um ano e sem anormalidades.

\section{DISCUSSÃO}

O tricobezoar ocorre em pacientes com transtornos mentais, com tricotilomania e tricofagia, sendo $90 \%$ em mulheres jovens ${ }^{2}$. O quadro clínico geralmente cursa com anorexia, perda de peso, dor ou plenitude epigástrica. Ocorrem vômitos, intolerância a alimentos sólidos e hematêmese ${ }^{3}$. Com o progressivo aumento do tricobezoar pode ser palpada massa abdominal alta. $\mathrm{O}$ diagnóstico pode ser comprovado por ultra-sonografia, seriografia contrastada e endoscopia digestiva alta ${ }^{1}$.

O tratamento do bezoar pode ser conservador, para os bezoares pequenos, com enzimáticos e procinéticos ${ }^{3}$; endoscópico (mais válido para fitobezoar e pouco útil para tricobezoar) ${ }^{1,3}$; tripsia eletrohidráulica ${ }^{1}$; cirurgia laparotômica; cirurgia videolaparoscópica ${ }^{2,4,5}$.

A cirurgia laparotômica supraumbilical permite através da gastrotomia uma abordagem direta e rápida. Porém, não há como eliminar o potencial de inconvenientes pós-operatórios imediatos desta abordagem e, principalmente a seqüela cicatricial inestética das incisões abdominais longitudinais.

A abordagem videolaparoscópica é de menor agressividade à parede abdominal. Porém, poderá apresentar dificuldade na utilização do coletor de resíduo cirúrgico, que ocorreu em nosso caso, em que não foi possível a inclusão total do resíduo, mesmo com a dimensão de 26x20 cm (2000 ml) do saco coletor utilizado. Isto determinou a necessidade de limpeza cuidadosa de toda a cavidade abdominal. A segunda dificuldade é a via de retirada do resíduo cirúrgico (tricobezoar), cuja opção foi através de mini-Pfannenstiel por ser de menor morbidade e sequiela cicatricial mínima.

As vantagens estéticas obtidas, em contraposição à infecção em dois portais de $5 \mathrm{~mm}$ e as demais de edema, derrame pleural e ascite (relacionadas ao estado nutricional) merecem reflexões.

Finalmente, a casuística de tratamento de tricobezoar por videolaparoscopia ainda é pequena e, somente com sua ampliação teremos uma posição mais definida no cenário de opções do tratamento desta afecção.

\begin{abstract}
Trichobezoars are gastric concretions of hair that sometimes extend throught the intestine. We report on a patient with trichobezoar managed by videolaparoscopic extraction, and discuss the advantages and disadvantages of this method, that can be the treatment of choice of this conditions.
\end{abstract}

Key Words: Bezoars; Video-assisted surgery.

\section{REFERÊNCIAS}

1. Wang YG, Seitz U, LiZY, et al. - Endoscopic management of huge bezoars. Endoscopy, 1998, 30(4):371-374

2. Nirasawa Y, Mori T, Ito Y, et al. - Laparoscopic removal of a large gastric trichobezoar. J Pediatr Surg, 1998, 33(4):663-665

3. De Barcker A, Van Nooten V, Vandenplas Y - Huge gastric trichobezoar in a 10-year-old girl: case report with emphasis on endoscopy in diagnosis and therapy. J Pediatr Gastroenterol Nutr, 1999, 28(5):513-515
4. Yao CC, Wong HH, Chen CC, et al. - Laparoscopic removal of large gastric phytobezoars. Surg Laparosc Endosc Percutan Techn, 2000, 10(4):243-245

5. Domene CE, Aun M, Volpe P. Extração de fitobezoar gástrico por videolaparoscopia : considerações técnicas originais. Rev Col Bras Cir, 2002, 29(4): 244-246

Endereço para correspondência:

Antonio Carlos Bartolomucci

Praça Julia Marcondes Salgado, nº 136

Jardim das Nações - Taubaté - SP - CEP: 12030-510

E-mail: acbartolo@bol.com.br 